\title{
Implicaciones de la argumentación en el aula de matemáticas
}

Implications of Argumentation in Math Class

Implicações da argumentação na sala de aula de matemáticas

Horacio Solar-Bezmalinovic* iD orcid.org/0000-0002-1958-8153

Artículo de investigación

Revista Colombiana de Educación, N.7 74. Primer semestre de 2018, Bogotá, Colombia.

Para citar este artículo: Solar, H. (2018). Implicaciones de la argumentación en el aula de matemáticas. Revista Colombiana de Educación, (74), 155-176.

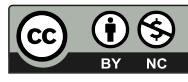

Recibido: 31/01/2017

Evaluado: 13/02/2017

* Doctor en Didáctica de las Matemáticas. Profesor asistente del Departamento de Didáctica. Facultad de Educación. Pontificia Universidad Católica de Chile. Santiago, Chile. Correo electrónico: hsolar@uc.cl 


\section{Resumen}

En este artículo de reflexión se presenta, mediante el estudio de casos de dos clases de matemáticas, tres implicaciones de promover la argumentación en el aula de matemá ticas: la primera es que por medio de una gestión argumentativa de la clase el profesor puede reconocer los patrones comunes de pensamiento en los estudiantes, la segunda es el uso de estrategias comunicativas del profesor para gestionar la clase generando una interacción dialógica entre profesor y estudiantes, y la tercera es que el docente puede contar con una mayor variedad de recursos para el abordaje de las contingencias en el aula de matemáticas. Las implicaciones de la argumentación muestran cómo el docente desarrolla recursos que contribuyen a generar criterios de flexibilidad ante la diversidad en el aula de matemáticas, para así superar las políticas de estandarización predominantes en el aula de matemáticas.

\section{Palabras clave}

argumentación matemática estrategias comunicativas; contingencia; justicia social

\section{Keywords}

mathematical reasoning; communicative strategies; contingency; social justice
This reflection paper presents, through the case study of two math classes, three consequences of fostering argumentation inside the math classroom: The first one is that, through an argumentative management of the class, the teacher can recognize common thinking patterns in students; the second one is the teacher's use of communicative strategies to manage the class by creating a dialogical interaction between teachers and students; and the third one is that teachers may have a wider variety of resources for addressing contingencies in the math classroom. The implications of argumentation show how teachers develop resources that contribute to generating flexibility criteria when faced with diversity in the math classroom, in order to overcome the predominant standardization policies in it

\section{Resumo}

Neste artigo de reflexão apresenta-se, por meio do estudo de casos de duas aulas de matemáticas, três implicações de promover a argumentação na sala de aula de matemáticas: a primeira é que por meio de uma gestão argumentativa da aula, o professor pode reconhecer os padrões comuns do pensamento nos estudantes; a segunda é o uso de estratégias comunicativas do professor para ministrar a aula, gerando uma interação dialógica entre professor e estudantes; e a terceira que o docente pode contar com uma maior variedade de recursos para a abordagem das contingências na sala de aula de matemáticas. As implicações da argumentação evidenciam como o docente desenvolve recursos que contribuem a gerar critérios de flexibilidade frente a diversidade na sala de aula de matemáticas, superando assim as políticas de estandardização predominantes.

\section{Palavras-chave}

argumentação matemática; estratégias comunicativas: contingência; justiça social 


\section{Introducción}

Los informes de resultados de los estudiantes en las pruebas estandarizadas internacionales PISA (Organización para la Cooperación y el Desarrollo Económicos, OCDE, 2013) muestran que los desempeños a nivel nacional y sudamericano están muy por debajo del estándar. Tal es el caso de matemática, en que Chile está 71 puntos bajo el promedio de la OCDE (Agencia de Calidad de la Educación, 2014). Sin embargo, las diferentes políticas educativas implementadas en el país no han logrado mejorar el aprendizaje profundo de los estudiantes en matemáticas. Uno de los factores que pueden explicar estos resultados negativos es la continuación de políticas de estandarización de la enseñanza que se centraron en la promoción de habilidades básicas en matemáticas (Mourshed, Chijioke y Barber, 2010). Hoy en día, en un enfoque por competencia (OCDE, 2013), estas políticas han dejado de tener sentido y es necesario repensar la educación matemática desde otra perspectiva. En este artículo de reflexión, pondré el acento en la argumentación como una competencia que contribuye a la justicia social en el aula.

\section{Competencias matemáticas}

El marco teórico de PISA (OCDE, 2013) ha sido un factor importante para que cada día sean más los países que orienten su currículo escolar en un enfoque por competencias. En particular, las Bases curriculares chilenas en matemáticas han incorporado cuatro habilidades: modelizar, argumentar y comunicar, representar y resolver problemas, las que deben desarrollarse en cada uno de los ejes de contenido (Mineduc, 2013). Si bien se puede esperar que este enfoque en las Bases curriculares sirva como fundamento para poder superar los bajos resultados en pruebas Como PISA, es necesario tener ciertas condiciones para el desarrollo de las competencias matemáticas, tales como profesores formados para ello y reconocer cuáles son las oportunidades de aprendizaje para los estudiantes en un enfoque por competencias.

El grupo de investigación Competencias Matemáticas (соммAт) ha venido realizando investigaciones sobre el desarrollo de las competencias matemáticas en el aula (Solar, Azcárate y Deulofeu, 2012; Solar, Deulofeu y Azcárate, 2015; Solar y Deulofeu, 2016). Una de las competencias en la que se ha puesto especial atención es la argumentación. En nuestras investigaciones hemos concluido que, por medio de un proceso de formación continua, los profesores son capaces de cambiar sus prácticas para promover la "argumentación en el aula de matemáticas", entendida como el intento de convencer o persuadir al otro en el aula de matemáticas; la diferenciamos de la "argumentación matemática", entendida como el proceso de prueba que enfrenta un resolutor ante una tarea matemática sin

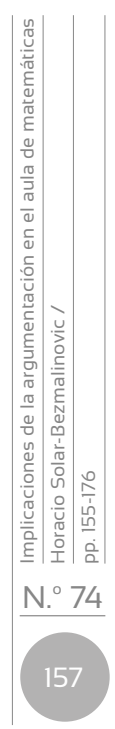


necesariamente confrontar dos puntos de vista (Solar y Deulofeu, 2016). Basándose en el modelo de argumentación de Toulmin (2003), algunos trabajos han estudiado la construcción individual de los argumentos en el aula de matemáticas, mientras que otros investigadores han destacado la argumentación colectiva como una parte importante del discurso en el aula de matemáticas, que incluye cualquier instancia en que el profesor y los estudiantes establecen una sentencia que se puede asociar a la argumentación (Krummheuer, 1995).

En un contexto en que profesores promueven la argumentación y en que se conocen condiciones para desarrollar la argumentación, se puede plantear la siguiente pregunta: ¿Qué oportunidades de aprendizaje tienen los estudiantes en un contexto de argumentación colectiva? La relevancia de la pregunta se justifica porque si bien existen varios marcos curriculares de matemáticas que consideran la argumentación como una competencia por promover en los estudiantes, y existe un conocimiento importante basado en investigaciones sobre argumentación matemática, las prácticas de argumentación de los estudiantes en el aula y su tratamiento en libros de texto sigue siendo insuficiente para lograr la intención de los marcos curriculares (Stylianides, Bieda, y Morselli, 2016).

\section{Argumentación en el aula de matemáticas}

Los análisis de argumentación en el aula se sustentan en el modelo argumentativo propuesto por (Toulmin, 2003), que sigue un proceso lineal desde los datos hasta las conclusiones. Esta secuencia consta de seis elementos (Goizueta y Planas, 2013): datos, conclusión, garantía, respaldo, calificador modal y refutadores.

Para estudiar la argumentación en el aula de matemáticas, Krummheuer (1995) propone una secuencia de argumentación basada en el modelo argumentativo de Toulmin. Sin embargo, reduce el sistema original a cuatro elementos: datos, garantía, respaldo y conclusión.

Diversas investigaciones sobre argumentación han seguido la reducción de Krummheuer en el uso del modelo argumentativo de Toulmin (Pedemonte, 2005; Rasmussen, Stephan y Allen, 2004) y han usado esta secuencia argumentativa en diversos temas matemáticos, tanto en primaria como en secundaria, mostrando que es útil transversalmente al nivel escolar y al contenido. Por otra parte, otros investigadores han indagado sobre la argumentación en la actividad matemática conservando todos los elementos del modelo argumentativo de Toulmin, destacando la necesidad de considerarlos todos para dar cuenta de la complejidad de tal actividad (Inglis, Mejia-Ramos, y Simpson, 2007). Desde la perspectiva de argumentación colectiva de Krummheuer (1995), Conner, Singletary, 
Smith, Wagner, y Francisco (2014) utilizan los seis elementos de la estructura de Toulmin y estudian el papel del profesor en el desarrollo de la argumentación, para ello modifican el modelo con el fin de incluir las acciones de los profesores que, si bien no están directamente asociadas a los componentes de la argumentación, contribuyen a su desarrollo. En nuestras investigaciones, hemos optado por esta última visión de la argumentación colectiva con los seis elementos de Toulmin en que los refutadores desempeñan un papel esencial para convencer o persuadir al otro en el aula de matemáticas.

Varios autores han puesto el foco en la importancia de los espacios de comunicación y discusión en el aula de matemáticas (Smith y Stein, 2011). En particular Lee (2006) señala diversas estrategias comunicativas que puede utilizar el profesor para incluir a todos los estudiantes en el discurso matemático, tales como dar oportunidades para aportar y asegurarles que las respuestas equivocadas revelan errores que el profesor necesita aclarar. Una primera condición que hemos detectado para promover la argumentación colectiva en el aula de matemáticas es el uso de este tipo de estrategias comunicativas por parte del profesor (Solar y Deulofeu, 2016), en que, por medio del fomento de la participación, la gestión del error, y preguntas deliberadas, el docente promueve el aprendizaje mediante una gestión argumentativa de la clase de matemáticas. Otras dos condiciones que hemos observado son: tareas matemáticas abiertas que permitan el contraste de posturas y una planificación de clases en que el docente anticipe la argumentación. Si en una clase se dan las tres condiciones para promover la argumentación colectiva, entendemos que el docente realiza una gestión argumentativa; en el caso de que no se aprecien tareas matemáticas abiertas ni una planificación de clases en que se anticipen distintas posiciones argumentativas, pero que aparezcan diferentes respuestas, es decir errores, procedimientos o posturas, de todos modos, puede darse una gestión argumentativa mediante un uso adecuado de las estrategias comunicativas.

\section{Contexto del estudio}

El estudio se realizó en el marco de un proyecto de investigación en que uno de los propósitos era identificar las condiciones para promover la argumentación en el aula de matemáticas; para ello se diseñó un seminario de profesores con miras a generar recursos en los docentes para promover la argumentación en el aula. En el seminario participaron diez docentes de enseñanza básica de establecimientos educacionales de la ciudad de Concepción (Chile) y tuvo una duración de 15 meses (2014-2015). El proceso se realizó bajo el modelo de formación Mejoramiento de la experiencia docente (Solar, Ortiz y Ulloa, en prensa), cuyo objetivo es que profesores en ejercicio estudien problemáticas en torno a la gestión 
del aula de matemáticas. En este modelo de formación para profesores de matemáticas, se promueve el desarrollo de la reflexión del profesor por medio del análisis de la práctica, utilizando grabaciones de clase.

En Solar y Deulofeu (2016) se describen el diseño metodológico para la recogida y análisis de los datos. En este artículo de reflexión, nos centraremos en las implicancias que tienen las clases en que se promueve la argumentación en la clase de matemáticas; para ello hemos seleccionado dos casos del estudio: el de Matilde (Solar y Deulofeu, 2016) y el de Mónica.

\section{Implicancias en la argumentación}

Producto de las investigaciones del grupo соммат, el desarrollo de la argumentación colectiva en el aula de matemáticas tiene tres implicancias interesantes de destacar: identificación de patrones de pensamiento, interacción dialógica entre profesor y estudiantes, y herramientas para abordar las contingencias. Finalmente, discutiremos cómo estas implicancias brindan oportunidades para la justicia social en educación matemática.

\section{Reconocimiento de patrones comunes de pensamiento en los estudiantes}

Si bien hay diferencias individuales y culturales entre los estudiantes, también se observan patrones comunes en las formas en que estos piensan y desarrollan comprensiones y habilidades en torno a determinados temas y problemas. Los profesores que están familiarizados con los patrones comunes de pensamiento y desarrollo de los estudiantes, y que tienen la habilidad para anticiparlos e identificarlos son capaces de trabajar en forma más efectiva y eficiente cuando planifican e implementan su enseñanza y cuando evalúan el aprendizaje de sus estudiantes. Este tipo de práctica se puede identificar en tres acciones del profesor en el aula: observar la forma de registrar y operar de los estudiantes, identificar errores comunes y proceder a su corrección, y reconocer ideas de sus estudiantes y organizarlas para precisar un concepto.

Los patrones de pensamiento pueden ser dificultades profundas de los estudiantes que pueden identificarse con obstáculos de origen epistemológico (Brousseau, 1983), en los que la noción de obstáculo es un conocimiento y no una falta de conocimiento (Duroux, 1982).

Las implicancias de la argumentación para abordar los patrones de pensamiento se han observado en un curso de primer año de secundaria (12-13 años) de un establecimiento educacional subvencionado de la ciudad de Concepción, en Chile. La profesora del curso, Matilde, tenía como propósito 
promover la argumentación en el aula. Si bien no había diseñado una clase para ello, a raíz de su participación en el seminario de profesores en que había visto videos de clases de otros docentes promoviendo argumentación en el aula, Matilde ya tenía ciertos conocimientos sobre cómo se desarrolla, por ejemplo la estructura de Toulmin y acciones que puede hacer el profesor para promover una argumentación colectiva entre los estudiantes.

En la clase se estudiaban los números enteros, y Matilde presentó el siguiente problema: "Un número entero y su inverso distan en la recta 12 unidades. ¿qué números son? Para ayudar a los estudiantes a comprender el enunciado, dibujó una recta numérica para situar los números y preguntó a los estudiantes cuáles son esos números, recibiendo como respuesta genérica -12 y 12 , en lugar de la respuesta esperada - 6 y 6 . La profesora en vez de evaluar la respuesta de los estudiantes para gestionar directamente el error, les hace leer nuevamente el problema y pregunta por el significado del término distan; varias de las respuestas de los estudiantes le dan como significado la distancia entre 0 y un número, en lugar de la distancia entre dos números cualesquiera. Ello puede explicar los valores dados de -12 y 12 , que están a doce unidades de distancia del 0 cada uno. Matilde, por medio de varias acciones docentes y de la utilización de la recta numérica, sigue trabajando con los estudiantes el significado de distan para contrastar la idea instalada de que la distancia se cuenta desde 0 hasta el número; entonces, emerge en uno de los estudiantes que el significado del término se asocia a la distancia entre dos números, y si se eligen - 12 y 12, se debe contar la distancia entre ambos números, que no es 12 sino 24 . En el siguiente episodio de la clase se muestra de qué manera Matilde gestiona estas ideas sobre el significado de distan.

Matilde: ¿Qué significa que diste 12 unidades?

Roberto: Que está distando 12 números ¿̇o no?

Matilde: ¿Cómo?

Arturo: La distancia que está del... la distancia que tiene que tener el 0 del...

Estudiantes: Hasta el 12.

Matilde: ¿Es la distancia del 0 hasta el 12?

Estudiantes: No... al -12... [murmullo de otras respuestas]

Matilde: ¿Cómo?

Martín: Del -12 hasta el...

Matilde: A ver, escuchemos... escuchemos a su compañero. Aquí tenemos otra idea, nosotros estamos centrados en que la distancia es del 0 hasta el 12, ¿qué dices tú?

Martín: Que... la distancia es del 12... del -12 hasta el 12. 
Matilde: Él me habla de que la distancia que me están hablando aquí, de 12 unidades, es de donde está [marca -12 en la recta]... hasta, y para acá [marca espacio hasta 12 en la recta] ¿Qué les parece a ustedes la opinión de él?

Estudiantes: Bien [tres estudiantes responden esto, el resto guarda silencio]

Matilde: $\quad$ O sea, ¿es del o desde... desde donde está uno hasta donde está el otro? ¿David?

David: $\quad$ Eeeh, es del 0 hasta donde está el otro porque de 12 hasta el otro daría 0.

Roberto: Daría 12 igual [Otros compañeros opinan, pero no se entiende lo que dicen ya que hablan todos juntos]

Mario: Daría 12 igual porque...

Matilde: ¿Francisco? ¿Escuchemos a Francisco?

Francisco: Empieza del -12 porque ahí dice que el número 12 y su inverso distan, no que el 0 dista...distan del 12.

Matilde: ¿Me lo explicas de nuevo? A ver, escuchemos a Francisco.

Francisco: Que ahí dice que el... el número entero 12 y su inverso distan, no del $0 \ldots$ eeeh, hasta el 12.

Matilde: ¿Se entiende? Eh, ¿Isabel?

Isabel: $\quad$ Pero ahí dice que la recta dista 12 unidades, y donde comienza la recta es donde el 0 , entonces sería el 0 .

Matilde para gestionar el error, en vez de evaluar la respuesta de los estudiantes realiza una gestión argumentativa para que sean ellos mismos quienes se den cuenta del error. Matilde contrapone dos posturas: estudiantes que responden que la distancia es entre 0 y 12 y otros que señalan que la distancia es entre -12 y 12 , e incentiva que el resto del curso opine sobre estas dos posturas. Al obtener solo tres respuestas, dirige una nueva pregunta a David respecto a desde qué punto de referencia se mide la distancia, y este sigue sosteniendo que desde el 0 al igual que Roberto y Mario, quienes lo apoyan. A continuación, Matilde le da la palabra a Francisco, quien es el primero en explicar por qué se mide la distancia desde -12 a 12 y asimismo refuta la idea instalada de 0 a 12 . Matilde, más que validar la intervención de Francisco, lo invita a seguir describiendo su idea para que el resto del curso lo atienda; en el curso sigue habiendo alumnas como Isabel, que continúan con la idea de que la distancia es entre 0 y 12, por lo que la profesora pide más opiniones y ante el silencio hace leer nuevamente el enunciado para dilucidar el significado de distan. 
La idea de Isabel de que la distancia es entre 0 y 12 evidencia el patrón de pensamiento de un sector importante de los estudiantes: la distancia se calcula a partir de 0; esta es la causa más probable del error de responder -12 a 12. Hasta ahora la respuesta de Matilde a la dificultad de los estudiantes es volver a leer el problema, lo cual no responde directamente al patrón de pensamiento sino más bien a una creencia por parte de Matilde de que el error se debe a una falta comprensión del problema. Pero mediante la gestión argumentativa, la profesora se comienza a dar cuenta de que este tiene relación con el significado de distancia en los números enteros que tienen los estudiantes.

En el siguiente episodio se aprecia cómo actúa Matilde una vez que ha reconocido la causa del error:

Matilde: Ya, ¿y qué distancia habría del -12, Daniel, al 12?

Estudiantes: 24.

A: No, 12 señorita.

Roberto: $\quad$ O sea no, sería 12.

Matilde: Tenemos...

Roberto: Porque del 0 se empieza a contar de nuevo.

A: Sí.

A: No.

Matilde: $\quad$ Ya, si de aquí hasta aquí tenemos una distancia de 12, ¿he llegado al inverso de -12? [Señala en la pizarra la distancia que hay de -12 hasta 0]

Estudiantes: No.

Matilde: Miren, voy de -12 a su inverso, avanzo, ¿ ¿cuánto llevo hasta aquí? [Marcando el 0 en la recta numérica]

Estudiantes: 12.

Matilde: Si sigo...

Javier: $\quad$ Es como multiplicarlo por 2 y sería 24.

Arturo: 24.

Matilde: ¿Cuánto avancé para llegar al inverso?

Estudiantes: 24.

Matilde: ¿Cuál es la distancia de -12 hasta 12 ?

Estudiantes: 24.

Matilde: Distan 24 unidades entre estos dos valores.

Estudiantes: Sería del -6 al 6 entonces. 
Para poder identificar de mejor manera la argumentación colectiva, se utilizará el esquema de Toulmin (véase la figura 1).

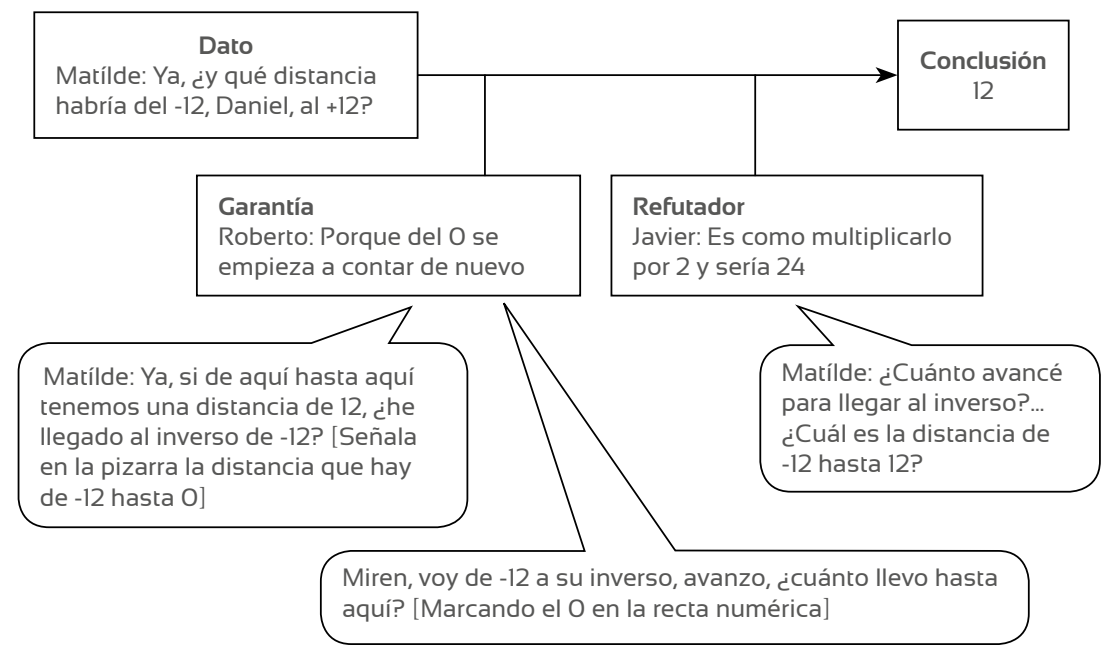

Figura 1. Mapa de la argumentación colectiva según el modelo de Toulmin (Solar y Deulofeu, 2016)

El dato corresponde a la pregunta de Matilde sobre cuál es la distancia entre -12 y 12 . Luego vienen dos respuestas que actúan como conclusiones diferentes: 24 y 12; para seguir el proceso argumentativo tomaremos como conclusión falsa el 12. Se sigue la estrategia de análisis de Conner et al. (2014), en que los cuadros corresponden a las intervenciones tanto de Matilde como de los estudiantes que son parte de la estructura argumentativa, en cambio en los globos se han puesto las intervenciones de Matilde, que contribuye por medio de preguntas a que aparezca la refutación a la falsa conclusión. Una mayor profundización del caso expuesto se puede encontrar en (Solar y Deulofeu, 2016).

Se puede apreciar que una vez que Matilde ha identificado el patrón de pensamiento que origina el error, por medio de la manipulación en la recta numérica y con acciones de avanzar desde el -12. hasta el 0 , los estudiantes van cambiando su idea y aprecian que sí se puede considerar la distancia en los números negativos, entre ellos Javier "Es como multiplicarlo por 2 y sería 24.", y de esta manera los estudiantes cambian su respuesta a -6 y 6

Una gestión argumentativa se puede utilizar para cualquier clase que tenga como propósito indagar en las ideas de los estudiantes. En el siguiente caso, relativo a la profesora Mónica, una clase para cuarto de primaria se ha diseñado para promover argumentación en clases. La profesora tenía 
como objetivo descubrir las regularidades en la multiplicación y división, y diseñó una tarea matemática para que los estudiantes encontrasen el número adecuado para que se cumpliera una igualdad.

En la figura 2 se muestra un extracto de la planificación de la clase para un cuarto de primaria. En la actividad A, la profesora Mónica espera que los estudiantes respondan de manera satisfactoria, escribiendo el 1 como el único número natural posible para que el resultado sea 1. En cambio, en la actividad B se esperan diferentes respuestas, incluidos varios errores asociados a la posición del cero. En la planificación se anticipan posibles errores, y si bien la profesora no anticipa las posibles garantías o refutaciones apoyándose en la estructura de Toulmin, tiene pensado abordar los errores a través de una gestión argumentativa.

Actividad A - ¿Qué números debe ir en el recuadro para que se cumpla la igualdad? ¿Cuántas posibilidades existen?

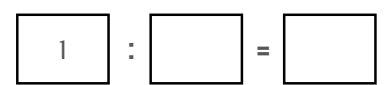

Actividad B - ¿Qué números deben ir en el recuadro para que se cumpla la igualdad? ?Cuántas posibilidades existen?

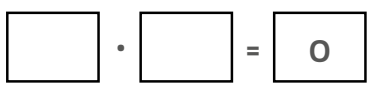

RESPUESTAS ESPERADAS

Para la actividad A se esperan que todas las respuestas sean correctas (1:1=1)

Para la actividad B se espara que surjan respuestas erróneas

Figura 2. Extracto del plan de clases de Mónica

En el transcurso de la clase, al desarrollar la actividad A aparecen diferentes respuestas erróneas de los estudiantes. La profesora Mónica hace pasar primero a la pizarra a los estudiantes que dan como resultado $1 \div 3=2$ y, mediante una gestión argumentativa, sin validar las respuestas va preguntando al curso si están de acuerdo con esta respuesta, a lo cual Tania responde: "No puede ser dos porque uno no se puede dividir en dos". Se siguen discutiendo diferentes resultados mediante una gestión argumentativa; entre estos aparece Isabel con otra respuesta: "A nosotros, con Javier, uno entre dos nos dio el resultado de dos", respuesta que también es gestionada por Mónica de manera argumentativa: 
Mónica: A ver Isabel, ustedes pusieron 1dividido en 2 es 2. ¿Por qué?

Solamente eso. Cuéntenos ¿por qué puso eso?

Isabel: $\quad$ Porque, con el Javier hicimos un ejemplo con una cajita y adentro habían [sic] dos galletas, y el resultado después le dio dos.

Mónica: Pase y explícanos [inicio cita] aquí con un dibujo.

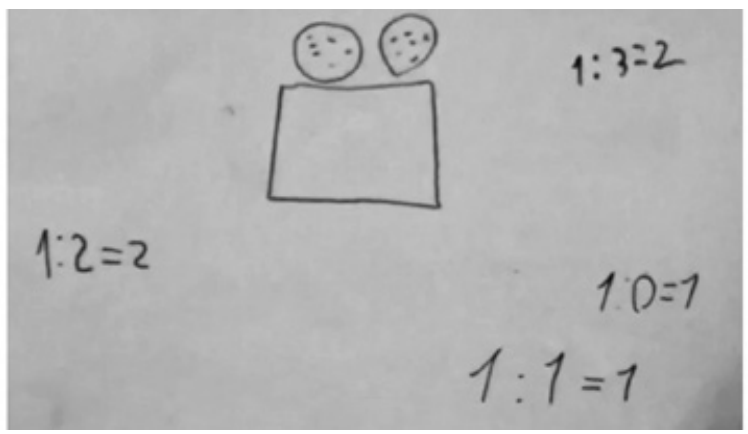

Figura 3. Múltiples respuestas a actividad A

Cuando Mónica solicitó a Isabel que explicara su respuesta, ella utilizó como ejemplo una caja con dos galletas para explicar el reparto, ante lo cual Mónica pidió que hicieran un dibujo de la idea de Isabel y Javier (véase la figura 3). Este ejemplo, que contiene un modelo de reparto equitativo para la división, es utilizado por otros estudiantes para justificar sus respuestas. Luego pasaron varios estudiantes a la pizarra a reproducir tres respuestas diferentes $(1 \div 3=2 ; 1 \div 2=2 ; 1 \div 2=1)$ abordadas por Mónica mediante una gestión argumentativa. Mónica se situó en el fondo de la clase, en la mesa de Valeria, para preguntarle su respuesta correcta $1 \div 1=1$.

Mónica: [Mónica está mirando a dos estudiantes que están en su asiento] ¿Y acá, qué tienen ustedes?

Valeria: uno dividido por uno es igual a uno

Mónica: ¿Distinto? ¿Lo pueden pasar a explicar?

[Valeria con su compañero pasan a la pizarra y escriben $1 \div 1=1$ ]

Mónica: Distinto. ¿Por qué ustedes pusieron 1?

Valeria: Dividimos uno por uno porque si nosotros tuviéramos una galletita y pusiéramos en una caja sería solamente uno porque teníamos solamente una galletita.

Mónica: Ya, aquí, tiene que haber alguien que tenga la razón. Porque dijimos que era una regularidad, algo que se da siempre, $y$ ahí tenemos muchas cosas. Ah, ya. ¿Qué cree usted?

Daniela: Que la Valeria está correcta.

Mónica: Que la Valeria está correcta. Usted estaba de acuerdo con otra persona. 
Daniela: Sí.

Mónica: ¿Con quién estaba de acuerdo antes?

Daniela: Con Tania.

Mónica: Con Tania y ahora, ¿Qué le hace cambiar de opinión?

Daniela: Porque uno es uno dividido en uno, y el uno solo se divide una sola vez.

Mónica: Uno dividido en uno es uno, porque el uno se divide una sola vez. ¿Eso era lo que usted estaba diciendo, o no? ¿Es lo mismo? Sí. Valeria, ¿Qué crees tú?

Mónica hace pasar a Valeria con su compañero a la pizarra para que expliquen el resultado y Valeria utiliza el modelo de la galleta para hacerlo. Mónica en vez de evaluar la respuesta para gestionar el error, mediante una gestión argumentativa pregunta al resto del curso quien puede tener la razón. Daniela señala que cambió de opinión y es ella misma quien refuta la respuesta de Tania, con quien estaba de acuerdo antes, señalando que "uno es uno dividido en uno, porque el uno se divide una sola vez".

Con esta respuesta se esperaría que la profesora ahora sí evaluara la respuesta de Valeria, pero en vez de eso, mantiene una gestión argumentativa y sigue preguntando a otros estudiantes sobre la respuesta. Como se aprecia en lo que sigue de la clase, cuando Mónica pregunta a otros estudiantes si cambiarían la opinión, Fernanda dice que cambia su respuesta de $1 \div 3=2$ a la dada por Isabel $(1 \div 2=2)$, quien sigue manteniendo su posición:

Mónica: Kevin, ¿qué cree ud.? Nada. Los que están ahí, todos los que están ahí... Eh, Fernanda, tienen uno dividido en tres es dos. Has escuchado a tus compañeros... ¿ Usted todavía cree que uno dividido en tres es dos, o cree que podría cambiar de opinión? ¿Podría cambiar de opinión? ...

Fernanda: Esa.

Mónica: Por esa [señala en la pizarra $1 \div 2=2$ ] uno dividido en dos es dos. ¿Y la Isabel? ¿Que tenía esa de las galletas? ¿Sigue pensando lo mismo o cambia de opinión?

Isabel: $\quad$ Sigo con lo mismo.

Mónica: Sigue pensando lo mismo...

La causa del error de Fernanda radica en que al realizar el reparto equitativo de una cantidad, ésta debe ser mayor que uno. Reconocemos en esta característica un posible patrón de pensamiento, puesto que aun habiendo aparecido la respuesta correcta y el correspondiente razonamiento que hay detrás de ella, aún se mantiene la posición de Isabel que 
es compartida, por lo menos, por Fernanda. Sin embargo, no hay suficiente evidencia que respalde esta posición, ya que la idea es declarada solo por dos estudiantes, que son quienes originan el error.

Mónica sigue indagando en la respuesta $1 \div 2=2$ para profundizar en el significado de los términos de la división, y para ello sigue con el modelo de repartir galletas, propuesto anteriormente por los estudiantes. Luego Mónica explicita que la multiplicación es la operación inversa de la división y mediante una revisión de las tres respuestas más recurrentes $1 \div 1=1$.

Hasta ahora se ha visto cómo promover la argumentación en clases se relaciona con que en ellas aparezcan patrones de pensamiento de los estudiantes que tienen relación con obstáculos, errores o ideas profundas. Una segunda implicancia que se destaca es la interacción dialógica entre profesor y estudiantes que se aprecia en una gestión argumentativa de la clase.

\section{Interacción dialógica entre profesor y estudiantes}

Desde el año 2010 el grupo commat ha participado en cursos de desarrollo profesional que han tenido como foco promover la argumentación en el aula de matemáticas. Estos cursos utilizan el modelo de formación Mejoramiento de la experiencia docente, que se centra en el análisis de la práctica del profesor mediante videos (Solar et al., en prensa). Los cursos han tenido una duración promedio de ocho meses, y se ha constatado cambios importantes tanto en su reflexión como en su desempeño en cuanto a la argumentación en clases (Solar y Deulofeu, 2016). Una de las maneras de apreciar estos cambios es mediante el uso de estrategias comunicativas por parte de los docentes.

En las clases de Mónica y Matilde se han analizado las tres estrategias comunicativas descritas en Solar y Deulofeu (2016): oportunidades de participación, gestión del error y tipo de preguntas, las que han sido claves para promover argumentación en sus clases.

La estrategia oportunidades de participación tiene la finalidad de asegurar que todos tengan la oportunidad de aportar. En el caso de Matilde una de las acciones más destacadas es no evaluar las respuestas de los estudiantes a lo largo de toda la clase, como podemos evidenciar de manera particular en el inicio del episodio en las preguntas que hace para promover la refutación, en vez de valorar la respuesta errónea de Roberto y quedarse con la respuesta inicial que dan algunos estudiantes antes de que aparezca la explicación de Roberto que hace de garantía. Otra acción destacada está asociada con la flexibilidad que tienen los estudiantes para intervenir en la discusión, lo cual también se aprecia a lo largo de toda la clase, y en particular en la variedad de estudiantes que intervienen. Matilde tiene la particularidad de ir relevando intervenciones clave, como la de 
Francisco, y da espacio a estudiantes como Isabel. Por otra parte, en la clase de Mónica la acción más destacada también tiene relación con no evaluar las respuestas de los estudiantes antes de socializar las respuestas, ello permite que se identifiquen los patrones de respuesta arraigados en los estudiantes, según los cuales el reparto debe ser en un número mayor a uno (por ejemplo $1 \div 2=2$ ).

Gestión del error. Esta estrategia tiene la finalidad de asegurar a los estudiantes que sus ideas/respuestas equivocadas son importantes para construir el conocimiento matemático. En la gestión de Matilde se destacan dos acciones: la primera es gestionar el error socializando de manera colectiva los conocimientos matemáticos, lo que permite ir mejorando la respuesta inicial; la segunda acción es no revisar en forma anticipada los errores, sino hasta después de que los estudiantes se han dado cuenta del error. Ambas acciones destacadas están muy presentes en el episodio, y son las que permiten que se genere la discusión entre las dos respuestas (12 y 24) y el proceso argumentativo descrito. En el caso de la clase de Mónica, se gestiona de manera argumentativa la multiplicidad de errores sin evaluar sus respuestas.

Tipo de preguntas. Esta estrategia pone el foco en la formulación de preguntas adecuadas por parte del docente, tales como preguntas que favorezcan la explicación, evitar preguntas retóricas, hacer contrapreguntas y preguntas que mantengan el foco en la discusión. En varias clases que se da la argumentación, se aprecia que las preguntas que mantienen el foco de la discusión favorecen especialmente el desarrollo de argumentación. En efecto, en los episodios de clase tanto de Matilde como de Mónica las interacciones entre las profesoras y los estudiantes en que las profesoras hacen preguntas para mantener el foco han sido cruciales para generar dos puntos de vista, lo que es fundamental para que exista argumentación.

Las tres estrategias comunicativas descritas son relevantes para gestionar la argumentación. En particular vemos que el tipo de preguntas es especialmente importante para la gestión especializada de la argumentación; en cambio, las otras dos estrategias sirven de apoyo para promoverla, ya que sin participación es difícil que aparezca argumentación, y la gestión del error promueve la contraposición de ideas.

\section{Contingencias en la clase de matemáticas}

La contingencia es un aspecto clave a la hora de analizar la gestión en el aula de matemáticas, tal como lo señalan Rowland, Huckstep y Thwaites (2005) dentro del marco teórico del Knowledge Quartet (KQ). En una clase puede haber muchas situaciones que son inesperadas y es el profesor quien toma la decisión, en ese mismo instante, de seguir con 
su planificación tal como está diseñada, o de tratar la nueva situación. Si bien esto puede ocurrir en cualquier tipo de clase, considero que en aquellas en que hay argumentación, estas situaciones de contingencia se pueden dar con mayor frecuencia, porque al incentivar que los estudiantes participen y que aparezcan diferentes posturas acerca de un resultado o procedimiento, el profesor favorece la aparición de muchas ideas de los estudiantes, algunas de las cuales pueden ser inesperadas. Ante ello, el profesor gestiona las situaciones de contingencia que se presentan en la búsqueda del desarrollo de la argumentación.

El Knowledge Quartet (KQ) (Rowland et al., 2005) es una teoría de base empírica sobre el conocimiento que debe tener un profesor, el cual se agrupa en cuatro dimensiones: fundamentación, transformación, conexión y contingencia. Esta última es la dimensión que se centra en las acciones del profesor en momentos imprevistos e inesperados. Se ha caracterizado la contingencia a partir de tres tipos de situaciones: ideas de los estudiantes, ideas del profesor y recursos del aula. Para la primera causa se han identificado tres tipos de desencadenantes: respuesta de los estudiantes a una pregunta del profesor; intervención espontánea de un estudiante o a una actividad o discusión, y respuestas incorrectas del estudiante (Rowland y Ruthven, 2011; Rowland, Thwaites, y Jared, 2015).

Ahora veremos cómo en las clases de Mónica y Matilde se presentan situaciones de contingencia producto de errores inesperados de los estudiantes.

Matilde planificó una clase que iniciaba con la actividad: "Un número entero y su inverso distan en la recta 12 unidades. ¿Qué números son?". Esta tarea matemática tenía como propósito activar conocimientos previos sobre la noción de valor absoluto que se utilizaría en otras tareas matemáticas de la misma clase. Matilde participaba del seminario de profesores y tenía intención de promover argumentación en esta clase, pero la tarea matemática señalada al ser de carácter introductorio no tenía intención de generar una variedad de respuestas, o diferentes posturas, y se esperaba generar argumentación en las tareas matemáticas siguientes; por ello el hecho de que gran parte de los estudiantes respondan -12 y 12 es inesperado para Matilde, es decir una contingencia desencadenada por un error inesperado de los estudiantes. La profesora, en vez de evaluar las respuestas, decide realizar una gestión argumentativa del error para que los propios estudiantes, por medio de una discusión de sus razonamientos, reconozcan el error. Matilde tenía planificada este tipo de gestión argumentativa para las siguientes tareas matemáticas y decidió modificar su plan para dedicarle gran espacio de la clase al desarrollo de esta tarea matemática inicial. 
En el caso de la clase de Mónica, la multiplicidad de respuestas de los estudiantes a la Actividad A (véase la figura 3), son inesperadas para la profesora. La respuesta $1 \div 3=2$ corresponde a una contingencia desencadenada por un error inesperado. Mónica realiza una gestión argumentativa en que aparecen nuevas respuestas que también son contingentes como la de Tania: "No puede ser dos, porque uno no se puede dividir en dos", y la de Isabel $1 \div 2=2$ asociada con el dibujo de la caja con dos galletas; pero seguramente la respuesta más inesperada para Mónica es que una vez que ya ha aparecido la respuesta correcta $1 \div 1=1$, Fernanda haya cambiado la suya de $1 \div 3=2$ a la de Isabel $(1 \div 2=2)$ quien mantiente su respuesta ante la pregunta de la profesora si cambiaba de opinión.

En estos dos casos, se aprecia cómo una gestión argumentativa hace que aparezcan diferentes situaciones de contingencia. Ahora bien, si quisiéramos determinar la calidad del abordaje de las contingencias por parte de las profesoras, las estrategias comunicativas que se utilizaron en el apartado anterior como una herramienta para identificar la calidad de la gestión argumentativa de la clase no permiten hacerlo, dado que ambas profesoras presentan una variedad de acciones docentes asociadas a las estrategias comunicativas, pero la calidad en el abordaje de las contingencias es muy distinta. Por ello se utilizarán otras dimensiones que corresponden a conjunto de habilidades fundamentales para la enseñanza denominadas High Leverage Practices (HLP) por el equipo de la Facultad de Educación de la Universidad de Michigan, o prácticas generativas, como las ha traducido el equipo de la Facultad de Educación de la Pontificia Universidad Católica de Chile. En el próximo apartado se analiza de qué manera abordar estas contingencias por medio de algunas prácticas generativas.

\section{Prácticas generativas para abordar las contingencias}

Las prácticas generativas ( $\mathrm{PG}$ ) son un conjunto de capacidades fundamentales para la enseñanza. El desarrollo adecuado de las prácticas generativas incrementa la posibilidad de que la enseñanza sea efectiva para el aprendizaje de los estudiantes (Ball, Sleep, Boerst, y Bass, 2009). En particular, considero que hay dos prácticas generativas de especial interés para una gestión argumentativa':

» PG 3 Suscitar e interpretar el pensamiento de cada estudiante: Los profesores formulan preguntas o tareas que animan a los estudiantes a compartir sus pensamientos acerca de contenidos académicos específicos, con el fin de poder evaluar su pensamiento, guiar discusiones instruccionales y destacar ideas que pueden beneficiar a otros estudiantes. Para identificar este tipo de práctica, decidimos

Se mantiene la numeración original de las 18 HLP. 
focalizarnos en tres tipos de acciones del profesor en el aula: elaborar preguntas que permitan al estudiante explicitar su pensamiento; chequear interpretaciones alternativas de las ideas y métodos de los estudiantes; y permitir distintas expresiones del pensamiento: lenguaje verbal (oral y escrito), simbólico o representaciones.

PG 5 Reconocer patrones particulares comunes del pensamiento de los estudiantes: Si bien hay diferencias individuales y culturales entre los estudiantes, también se observan patrones comunes en la forma en que los estudiantes piensan y desarrollan comprensiones y habilidades en torno a determinados temas y problemas. Los profesores que están familiarizados con los patrones comunes de pensamiento y desarrollo de los estudiantes y que tienen la habilidad para anticiparlos e identificarlos son capaces de trabajar en forma más efectiva y eficiente cuando planifican e implementan su enseñanza y cuando evalúan el aprendizaje de sus estudiantes. Esta PG corresponde a la primera implicancia en la argumentación, presentada como reconocimiento de patrones de pensamiento. Ya se han descrito algunas acciones del profesor asociadas, tales como que el profesor observe formas de registrar y operar de los estudiantes; que identifique errores comunes y proceda a su corrección; y que reconozca ideas de sus estudiantes y las organice para precisar un concepto.

Desde el punto de vista de las prácticas generativas, en la clase de Matilde se aprecia que la profesora, ante la respuesta inesperada de los estudiantes, -12 y 12, realiza una gestión argumentativa por medio de preguntas para que los estudiantes expliciten su pensamiento, tales como "¿Qué significa que diste 12 unidades?"; se detiene en las ideas alternativas con intervenciones como " Es la distancia del 0 hasta el 12?"... "A ver, escuchemos... escuchemos a su compañero. Aquí tenemos otra idea, nosotros estamos centrados en que la distancia es del 0 hasta el 12, ¿qué dices tú?". Asimismo, en los episodios mostrados se aprecia que continuamente va permitiendo la expresión del pensamiento oral, reflejado en la interacción con Arturo, David, Francisco e Isabel. Entonces podemos afirmar que Matilde mediante estas acciones ha suscitado e interpretado el pensamiento de los estudiantes. Ello tiene como consecuencia que pueda establecer un patrón común de pensamiento, que tiene relación con identificar que varios estudiantes no están considerando la distancia en los números negativos en la recta numérica y comienzan a contar desde el 0 , es por ello que para la distancia entre -12 y 12 obtienen 12 . Ello se ve reflejado en las siguientes acciones por parte de Matilde: ha identificado los errores de los estudiantes; reconoce las ideas detrás del error de -12 y 12 y las organiza en las condiciones para tomar la distancia en la recta numérica con números enteros. 
Por tanto vemos que la contingencia que ha aparecido (respuesta inesperada de - 12 y 12) ha sido gestionada mediante la PG 3 de suscitar e interpretar y la PG 5 de reconocimiento del patrón de pensamiento.

En la clase de Mónica, en los episodios descritos se pueden apreciar varias acciones de la profesora que se asocian a la PG 3, tales como elaborar preguntas que permiten que el estudiante expliciten lo que piensan, y permitir la expresión del pensamiento por medio del dibujo de la caja con la galleta. Se considera que estas suscitan el pensamiento de los estudiantes Pese que Mónica realiza este tipo de acciones, no profundiza en los razonamientos detrás del dibujo de la caja de galletas de los estudiantes, por tanto no interpreta sus ideas. En consecuencia, logra de manera parcial las estrategias asociadas a la PG 3.

Respecto a la PG 5, anteriormente se señaló que se aprecia un patrón de pensamiento de los estudiantes al interpretar la división como un reparto equitativo que debe ser de una cantidad mayor que uno. Si bien aparece la respuesta correcta de $1 \div 1=1$ y el correspondiente razonamiento que hay detrás de ella, aún se mantiene la posición de Isabel que es compartida, por lo menos, por Fernanda $(1 \div 2=2)$.

Se puede apreciar que Mónica ha realizado diferentes acciones para identificar los patrones de pensamiento: ha observado las maneras de operar, ha identificado los errores y, mediante una gestión argumentativa, ha procedido a corregirlos. Sin embargo, no ha reconocido la idea de reparto que hay detrás de la respuesta errónea $1 \div 2=2$. Por tanto, si bien Mónica cumple algunas acciones, no identifica completamente los patrones de pensamiento.

\section{Discusión y conclusiones}

A partir de las diferentes propuestas didácticas que tienen por finalidad mejorar la participación y los resultados de estudiantes para promover la inclusión en el aula de matemáticas (Valero, 2017), considero que la argumentación entendida como colectiva y necesariamente asociada a la idea de enfrentar dos puntos de vista (Solar y Deulofeu, 2016) tiene una incidencia directa en el desarrollo de la justicia social en el aula de matemáticas. Las tres implicancias de promover la argumentación que se han presentado (reconocimiento de patrones de pensamiento, interacción dialógica entre profesor y estudiantes, y contingencias en la clase de matemáticas), evidenciadas en los dos casos analizados, muestran la importancia que tiene la argumentación para superar las políticas de estandarización (Mourshed et al., 2010). En este sentido quiero destacar que las situaciones de contingencia constituyen oportunidades para profundizar el aprendizaje de los estudiantes a partir de errores que posiblemente son difíciles de tratar en la clase sin desviarse de la planificación. Por tanto, ante la necesidad de desviar la agenda planeada, 
la gestión argumentativa ofrece herramientas al profesor para abordar con eficiencia lo inesperado, y a su vez, criterios de flexibilidad que permiten superar las políticas de estandarización mencionadas.

Una manera de lograr justicia social es promover oportunidades de aprendizaje similares para todos los estudiantes. Se puede favorecer que esto ocurra por medio de una clase dialógica, en que el docente pueda ser flexible y aborde las contingencias que se presenten, para que así pueda reconocer y proponer estrategias a partir de los patrones de pensamiento de los estudiantes.

Para lograr justica social se requiere poner el foco más en la formación continua de profesores que en la formación inicial, ya que son temas que pueden ser trabajados a mediano plazo con los docentes. Tal ha sido nuestra experiencia, en que en menos de un año hemos visto cambios importantes en los profesores que han participado en los cursos de formación en temas como la argumentación en la clase de matemáticas².

\section{Referencias}

Agencia de Calidad de la Educación. (2014). Informe Nacional Resultados Chile PISA 2012. Recuperado de http://www.agenciaeducacion. cl/estudios-e-investigaciones/estudios-internacionales/PISA-programme-for-international-student-assessment/\%0D

Ball, D. L.; Sleep, L.; Boerst, T. A. y Bass, H. (2009). Combining the Development of Practice and the Practice of Development in Teacher Education. Elementay School Journal, 109(5), 458-474.

Brousseau, G. (1983). Les obstacles épistémologiques et les problèmes en mathématiques. Recherches En Didactique Des Mathématiques, 4(2), 165-198.

Conner, A. M.; Singletary, L. M.; Smith, R. C.; Wagner, P. A. y Francisco, R. T. (2014). Teacher support for collective argumentation: A framework for examining how teachers support students' engagement in mathematical activities. Educational Studies in Mathematics, 86(3), 401-429. Recuperado de https://doi.org/10.1007/s10649-014-9532-8.

Duroux, A. (1982). La valeur absolue: difficult és majeures pour une notion mineure. Burdeos: Publications de l'IREM.

Goizueta, M. y Planas, N. (2013). Temas emergentes del análisis de interpretaciones del profesorado sobre la argumentación en clase de matemáticas. Enseñanza de las Ciencias, 31(1), 61-78.

2 Más información sobre nuestro trabajo en formación continua en www.experienciadocente.uc.cl. 
Inglis, M.; Mejia-Ramos, J. P. y Simpson, A. (2007). Modelling mathematical argumentation: The importance of qualification. Educational Studies in Mathematics, 66(1), 3-21. Recuperado de https://doi.org/10.1007/ s10649-006-9059-8.

Krummheuer. (1995). The ethnography of argumentation. En P. Cobb y H. Bauersfeld (ed.). The emergence of mathematical meaning: Interaction in classroom cultures (pp. 229-269). Hillsdale, NJ: Lawrence Erlbaum.

Lee, C. (2006). Language for learning mathematics: Assessment for learning in practice. Inglaterra: Open University Press.

Mourshed, M.; Chijioke, C. y Barber, M. (2010). How the world's most improved school systems keep getting better. Nueva York: McKinsey y Company.

Organización para la Cooperación y el Desarrollo Económicos, OCDE. (2013). PISA 2012 Assessment and analytical framework: Mathematics, Reading, Science, Problem Solving and Financial Literacy. Recuperado de https://doi.org/http://dx.doi.org/10.1787/9789264190511-en.

Pedemonte, B. (2005). Quelques outils pour I'analyse cognitive du rapport entre argumentation et démonstration. Recherches En Didactique Des Mathématiques, 25(3), 313-348.

Rasmussen, C.; Stephan, M. y Allen, K. (2004). Classroom mathematical practices and gesturing. Journal of Mathematical Behavior, 23(3), 301 323. Recuperado de https://doi.org/10.1016/j.jmathb.2004.06.003.

Rowland, T.; Huckstep, P. y Thwaites, A. (2005). Elementary teachers' mathematics subject knowledge: The knowledge quartet and the case of Naomi. Journal of Mathematics Teacher Education, 8(3), 255281. Recuperado de https://doi.org/10.1007/s10857-005-0853-5.

Rowland, T.; Thwaites, A. y Jared, L. (2015). Triggers of contingency in mathematics teaching. Research in Mathematics Education, 17(2), 74-91. Recuperado de https://doi.org/10.1080/14794802.2015.1018931.

Rowland, T. y Ruthven, K. (2011). Mathematical knowledge in teaching. Londres/Nueva York: Springer.

Smith, M. S. y Stein, M. K. (2011). 5 practices for orchestrating mathematics discussion. EEUU: NCTM.

Solar, H.; Azcárate, C. y Deulofeu, J. (2012). Competencia de argumentación en la interpretación de gráficas funcionales. Enseñanza de las Ciencias, 30(3), 133-154.

Solar, H.; Deulofeu, J. y Azcárate, C. (2015). Modeling competence in functional graph interpretation. Enseñanza de las Ciencias, 33(2), 191-210. Recuperado de https://doi.org/http://dx.doi.org/10.5565/rev/ensciencias. 1551 
Solar, H.; Ortiz, A. y Ulloa, R. (2017). Modelo de formación continua para profesores de matemática, basada en la experiencia. Estudios Pedagógicos.

Solar, H. y Deulofeu, J. (2016). Conditions to promote the development of argumentation competence in the mathematics classroom. Bolema-Mathematics Education Bulletin, 30(56), 1092-1112. Recuperado de https://doi.org/10.1590/1980-4415v30n56a13.

Stylianides, A. J.; Bieda, K. N. y Morselli, F. (2016). Proof and argumentation in mathematical education research. En Á. Gutiérrez, G. C. Ledel y P. Boero (eds.). The Second handbook of research on the psychology of mathematics education (pp. 315-351). Rotterdam: Sense. Recuperado de https://doi.org/10.1007/978-94-6300-561-6_9.

Toulmin, S. E. (2003). The uses of argument. Cambridge: Cambridge University Press. Recuperado de https://doi.org/10.2307/2183556.

Valero, P. (2017). El deseo de acceso y equidad en la educación matemática. Revista Colombiana de Educación, (73), 99-128. 